\title{
Tandem Mass Spectrometry for the Determination of the Sites of DNA Interstrand Cross-Link
}

\author{
Yuesong Wang, Qingchun Zhang, and Yinsheng Wang \\ Department of Chemistry, University of California at Riverside, Riverside, California, USA
}

\begin{abstract}
Formation of DNA interstrand cross-link is implicated in the mechanism of anticancer activity of some drugs. Here we examined the fragmentation of deprotonated ions of double-stranded oligodeoxynucleotides (ODNs) that are covalently held together with either a mitomycin $C$ or a 4,5',8-trimethylpsoralen. Our results showed that, upon collisional activation, the covalentlybound duplex ODNs cleaved to give a series of $\mathrm{w}_{\mathrm{n}}$ and $\left[\mathrm{a}_{\mathrm{n}}\right.$ - base] ions; the sites of interstrand cross-linking could be determined from the mass shifts of some product ions. In addition, compared with the product-ion spectra acquired on an ion trap, those obtained from sustained off-resonance irradiation-collisionally activated dissociation (SORI-CAD) on a Fourier transform mass spectrometer offered high mass-resolving power, which facilitated unambiguous assignment of product ions and made it an effective method for locating the cross-linking sites. (J Am Soc Mass Spectrom 2004, 15, 1565-1571) (c) 2004 American Society for Mass Spectrometry
\end{abstract}

$\mathrm{D}$ NA interstrand cross-linking (ICL) agents are among the most powerful agents for cancer treatment $[1,2]$. In this context, psoralen [3] forms a cross-link between the two thymines at $5^{\prime}-\mathrm{TpA}-3^{\prime}$ site in opposite DNA strands upon irradiation with 365-nm UV light [3] (Scheme 1). Mitomycin C (MC), on the other hand, binds covalently to two guanine residues at $5^{\prime}-\mathrm{CpG}-3^{\prime}$ site in opposing DNA strands after reductive activation in vitro or in vivo [4] (Scheme 1). To develop new interstrand cross-linking drugs and to understand the mechanism of their anticancer activity, we need to have a sensitive method for locating the site and elucidating the structure of the interstrand cross-link. To attain the former goal, Hopkins and coworkers $[5,6]$ reported a method where they separated singly end-radiolabeled, singly crosslinked DNA from unreacted and monoalkylated singlestranded DNA by denaturing polyacrylamide gel electrophoresis (PAGE) followed by random cleavage with iron(II)/EDTA. However, radio-labeling and PAGE separation are necessary, which make the method inconvenient and labor-intensive.

Recently we reported a coupled enzymatic digestion/tandem MS (MS/MS) method for the structure elucidation of duplex oligodeoxynucleotides (ODNs) bearing an interstrand cross-link [7]. In that method we degraded the cross-linked duplex ODNs to smaller fragments containing the cross-linked nucleosides with

Published online September 18, 2004

Address reprint requests to Dr. Y. Wang, Department of Chemistry-027, University of California at Riverside, Riverside, CA 92521-0403, USA. E-mail: yinsheng.wang@ucr.edu an endonuclease, nuclease P1, and subjected these fragments to ESI-MS and MS/MS analyses. Our results from that study showed that MS/MS provides structure information of the cross-link. It can even allow us to differentiate the two orientation isomers of the MC interstrand cross-link [7].

Herein we report the application of MS/MS on a Fourier-transform ion cyclotron resonance (FT-ICR) and an ion-trap mass spectrometer for the determination of the sites of interstrand cross-link. We chose sustained off-resonance irradiation collisionally-activated dissociation (SORI-CAD) on the FT-ICR because of FTICR's ultrahigh resolving power and high mass accuracy [8, 9]. Two commonly used interstrand cross-linking agents, MC and 4,5',8-trimethylpsoralen (TMP), were employed to react with duplex ODNs to generate interstrand cross-linking complex. Upon collisional activation by SORI, cleavages within either of the two covalently-bound strands give a series of $\mathrm{w}_{\mathrm{n}}$ and $\left[\mathrm{a}_{\mathrm{n}}-\right.$ base] ions. When the fragmentation of unmodified single-stranded ODN and that of interstrand crosslinked ODN were compared, much larger masses for some fragments were observed for the latter. The sites of interstrand cross-linking can be readily determined from the mass shifts of some product ions. We also acquired product-ion spectra of the interstrand crosslinked ODNs on an ion-trap mass spectrometer. It is difficult, however, to determine the charge states for large product ions due to ion trap's low resolving power. We found that SORI-CAD on a FT-ICR mass spectrometer is an effective method for the determination of the sites of interstrand cross-linking. 


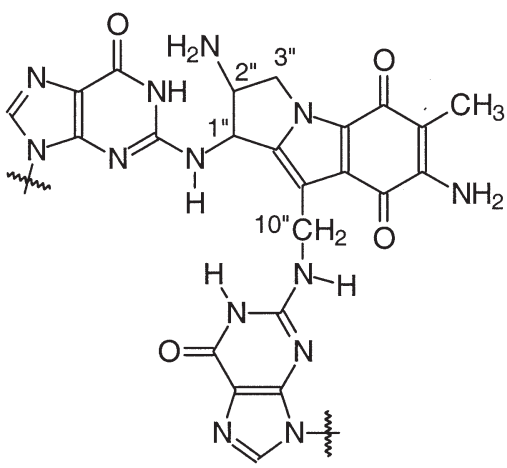

MC-Gua ICL

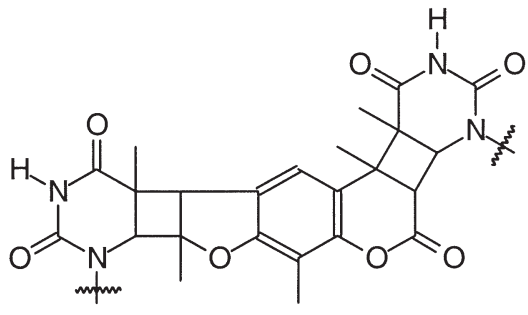

TMP-Thy ICL

Scheme 1. Structures for the two interstrand cross-links discussed in this study: interstrand cross-link of mitomycin $C$ with guanine (MC-Gua ICL) and interstrand cross-link of 4,5',8-trimethylpsoralen with thymine (TMP-Thy ICL).

\section{Experimental}

\section{Reagents}

ODNs used in this study were purchased from Integrated DNA Technologies, Inc. (Coralville, IA) and used without further purification. Mitomycin $C$ was obtained from A. G. Scientific Inc. (San Diego, CA), and all other chemicals were from Sigma-Aldrich (St. Louis, MO).

\section{Preparation of DNA ICL Products}

We prepared duplex ODNs containing a TMP interstrand cross-link following a procedure reported by Yeung and coworkers [10]. Briefly, a 250-nmol self-complementary ODN d(CGCGCTAGCGCG) was dissolved in 450- $\mu \mathrm{L}$ reaction buffer, which contained $0.2 \mathrm{mM}$ EDTA, $50 \mathrm{mM}$ $\mathrm{NaCl}$, and $5 \mathrm{mM}$ Tris (pH 7.6); the ODN was annealed by heating to $90-95^{\circ} \mathrm{C}$ followed by cooling slowly to room temperature over $3 \mathrm{~h}$. The ODN solution was then diluted to $100 \mathrm{~mL}$ with the reaction buffer, to which $1-\mathrm{mL}$ saturated TMP solution in ethanol was added. The resulting solution was dispersed in three 10.4-cm ID petri-dishes and irradiated on ice for $40 \mathrm{~min}$ with two 15-W Spectroline light tubes emitting at $365 \mathrm{~nm}$ (Spectronics Corporation, Westbury, NY). The distance between the light tubes and the petri dishes was approximately $3 \mathrm{~cm}$. The irradiation mixture was dried by using a Savant Speed-Vac (Savant Instruments Inc., Holbrook, NY) and the dried residue was redissolved in doubly-distilled water and separated by HPLC.

The mitomycin $C$ interstrand cross-link was prepared following a method reported by Tomasz and coworkers [11] with some modifications. A 50-nmol self-complementary ODN d(ATATACGTATAT) was dissolved in 1.2-mL of the above reaction buffer and the ODN was annealed in a similar way as described above. The ODN solution was incubated in an ice-water bath and mitomycin $C$ was then added to the solution at a molar ratio of $48 / 1$ (MC/duplex $\mathrm{ODN}$ ). Subsequently five portions of 0.4-M freshly prepared, ice-cooled, deareated $\mathrm{Na}_{2} \mathrm{~S}_{2} \mathrm{O}_{4}$ solution in the reac- tion buffer was added to the ODN/MC mixture at 10-min intervals (The molar ratio between $\mathrm{Na}_{2} \mathrm{~S}_{2} \mathrm{O}_{4}$ and $\mathrm{MC}$ was 1.5 to 1). The solution was under continuous argon bubbling during reaction and the reaction was terminated by exposing the mixture to air. The ICL-bearing duplex ODN was again isolated by reverse-phase HPLC as described below. The MC-ICL-containing, non-self-complementary duplex ODNs were prepared in a similar way.

\section{HPLC Conditions}

The HPLC separation was performed on a system composed of a Hitachi L-6200A pump (Hitachi Ltd., Tokyo, Japan) and a SSI 500 variable wavelength UV detector (Scientific System Inc., State College, PA). A 4.6 $\times 250 \mathrm{~mm}$ YMC ODS-AQ column (5 $\mu \mathrm{m}$ in particle size and $120 \AA$ in pore size, Waters Co., Milford, MA) was used. The two mobile phases were a solution of $50 \mathrm{mM}$ TEAA (Solution A) and a mixture of $50 \mathrm{mM}$ TEAA and acetonitrile (70/30, vol/vol, Solution B). A gradient of 5 $\min 0-20 \%$ B, $65 \min 20-50 \%$ B, and $25 \min 50-80 \%$ B was employed and the flow rate was $0.8 \mathrm{~mL} / \mathrm{min}$. The column temperature was maintained at $35^{\circ} \mathrm{C}$.

\section{Mass Spectrometry}

Most experiments were performed on a 4.7-T Fouriertransform mass spectrometer (IonSpec Co., Lake Forest, $\mathrm{CA})$ in the negative-ion mode. Ions were produced by electrospray ionization with an Analytical source (Analytical of Branford, Branford, CT) and accumulated in a storage hexapole for 1.2-2.0 s prior to injection into ICR cell by a quadrupole ion guide. Samples were dissolved in $\mathrm{CH}_{3} \mathrm{CN} / \mathrm{H}_{2} \mathrm{O}(1 / 1, \mathrm{vol} / \mathrm{vol})$ at a concentration of $5 \mu \mathrm{M}$ and infused with a syringe pump at a flow rate of 1 $\mu \mathrm{L} / \mathrm{min}$. For MS/MS experiments, precursor ions of interest were isolated in the ICR cell by using an arbitrary waveform generator followed by activation through sustained off-resonance irradiation (SORI) [12, 13]. Optimal results were obtained by irradiation frequencies that were 


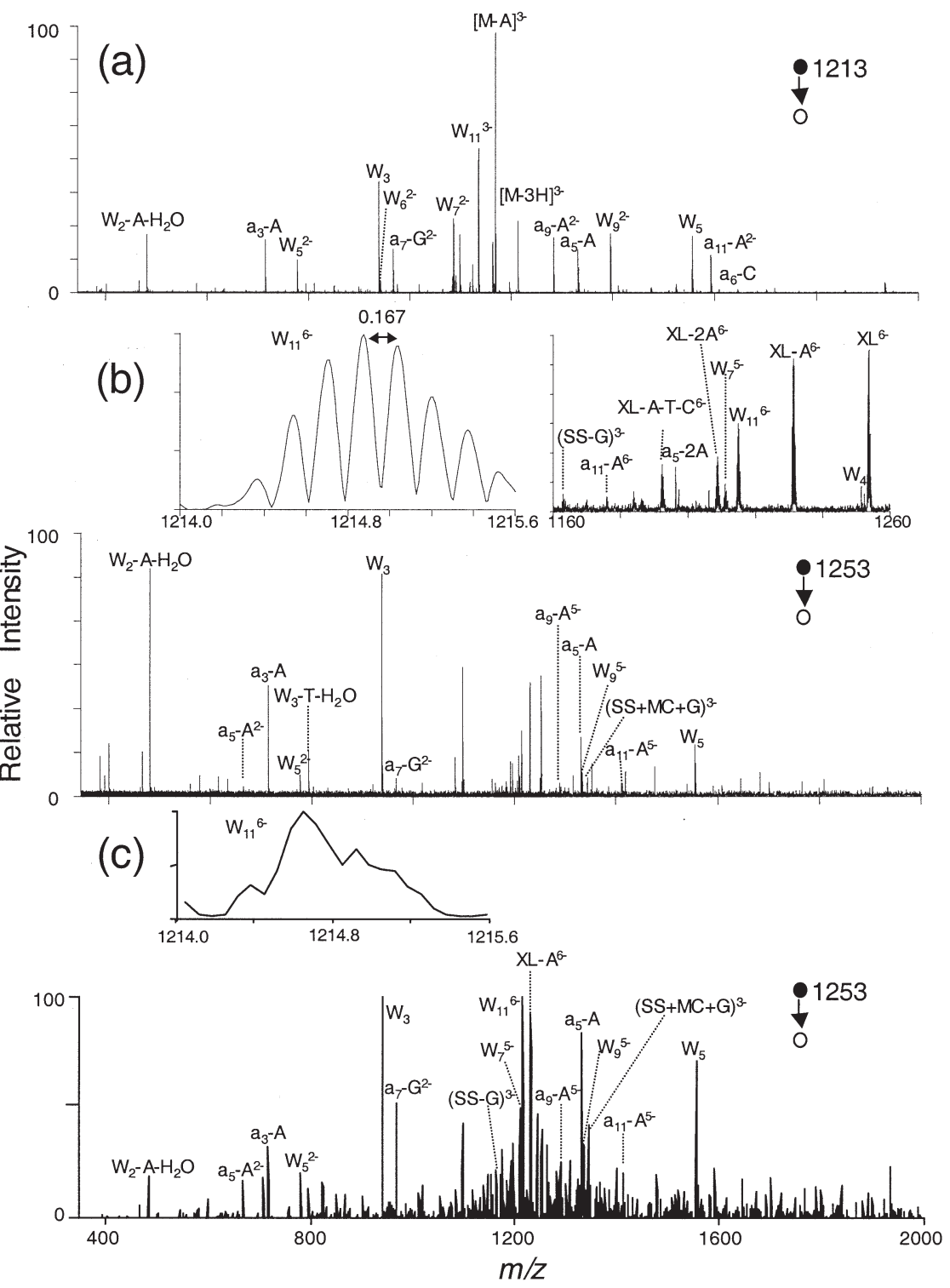

Figure 1. (a) Product-ion spectra of the ESI-produced $[\mathrm{M}-3 \mathrm{H}]^{3-}$ ion $(\mathrm{m} / \mathrm{z} 1213)$ of single-stranded d(ATATACGTATAT) (a) and $[\mathrm{M}-6 \mathrm{H}]^{6-}$ ion $(\mathrm{m} / \mathrm{z}$ 1253) of double-stranded, self-complementary d(ATATACGTATAT) cross-linked with MC (b). Both spectra were acquired by SORI-CAD on an FT-ICR mass spectrometer. (c) Same as in (b) except that the spectrum was acquired on an ion trap. "XL" and "SS" represent ICL-bearing and single-stranded ODNs, respectively. All nucleobases in fragment ions in this and other figures are represented by their single-letter codes.

600-1000 Hz lower than the cyclotron frequencies of the precursor ion. SORI-CAD of the analyte ion was performed for $1000 \mathrm{~ms}$ by a r.f. burst with an amplitude varied from 1.2 to $2.0 \mathrm{~V}$ depending on the analytes. Nitrogen was used as collision gas in all SORI-CAD experiments. After a delay of $2 \mathrm{~s}$, the resulting fragment ions were accelerated for detection by a r.f. sweep excitation waveform $\left(100 \mathrm{~V}_{p-p}\right)$. The image current was amplified, digitized at an acquisition rate of $2 \mathrm{MHz}$, and Fourier transformed to yield a mass spectrum.

Product-ion spectra for some ODNs were also acquired on an LCQ Deca XP ion-trap mass spectrometer (ThermoFinnigan, San Jose, CA). An equal-volume sol- vent mixture of acetonitrile and water was used as carrier solvent for electrospray, and a $2-\mu \mathrm{L}$ aliquot of sample solution $(\sim 5 \mu \mathrm{M})$ was injected in each run. The spray voltage was $3.4 \mathrm{kV}$, and the capillary temperature was maintained at $200{ }^{\circ} \mathrm{C}$.

\section{Results and Discussion}

\section{Fragmentation of Duplex ODNs Containing} a Mitomycin C Interstrand Cross-Link

To simplify the interpretation of product-ion spectra, we began with a self-complementary ODN, d(ATAT- 

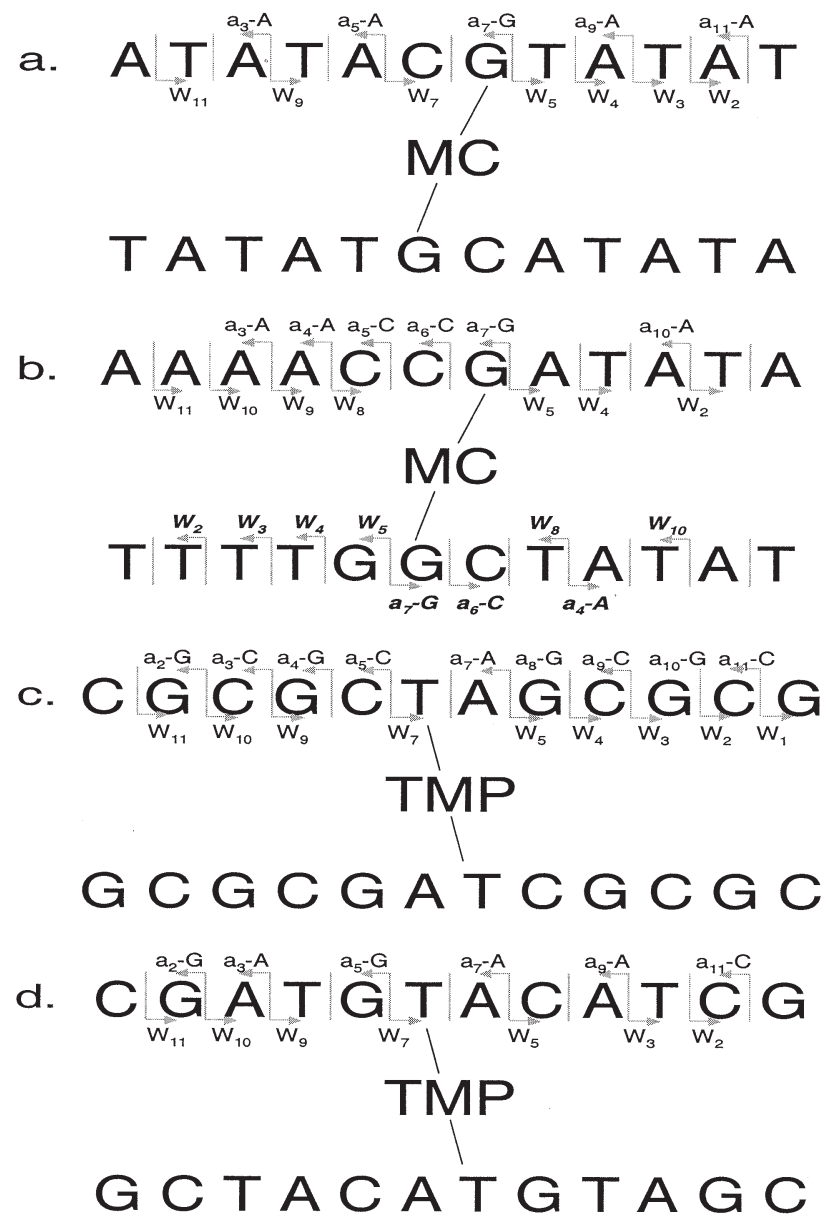

Scheme 2. A summary of the $\mathrm{w}_{\mathrm{n}}$ and $\left[\mathrm{a}_{\mathrm{n}}-\right.$ base] ions observed in the SORI-CAD spectra of ICL-bearing ODNs.

ACGTATAT). The product-ion spectrum of the [M $6 \mathrm{H}]^{6-}$ ion $(m / z$ 1253) of MC-ICL-bearing ODN (Figure $1 b$ ) showed the formation of $w_{n}$ ions, i.e., $w_{2}, w_{3}, w_{4}$, $\mathrm{w}_{5}{ }^{2-}, \mathrm{w}_{7}{ }^{5-}, \mathrm{w}_{9}{ }^{5-}$, and $\mathrm{w}_{11}{ }^{6-}$, and $\left[\mathrm{a}_{\mathrm{n}}-\right.$ base] ions, i.e., $\left[\mathrm{a}_{3}-\right.$ Ade], $\left[\mathrm{a}_{5}-\mathrm{Ade}\right],\left[\mathrm{a}_{7}-\mathrm{Gua}\right]^{2-},{ }^{2}, \mathrm{a}_{9}-\mathrm{Ade}^{5-}{ }^{5}$, and $\left[\mathrm{a}_{11}-\mathrm{Ade}\right]^{5-}$ ions ( "Ade" and "Gua" represent adenine and guanine, respectively, Scheme $2 \mathbf{a}$, nomenclature for fragment ions follows that reported by McLuckey et al. [14]). Among those product ions, the $\mathrm{w}_{7}, \mathrm{w}_{9}, \mathrm{w}_{11},\left[\mathrm{a}_{9}>\right.$ Ade $]$, and $\left[\mathrm{a}_{11}-\right.$ Ade $]$ ions from the interstrand cross-linked ODN showed much higher masses than the corresponding fragments formed from the collisional activation of the $[\mathrm{M}-3 \mathrm{H}]^{3-}$ ion of the unmodified single-stranded ODN (MS/MS of the [M $3 \mathrm{H}]^{3-}$ ion is shown in Figure 1a). The mass shifts of those fragment ions are consistent with that they bear not only the remnant of the strand being cleaved but also the MC and the opposing single strand. On the other hand, we found that all other product ions, including the $\mathrm{w}_{5}$ and $\left[\mathrm{a}_{7}-\mathrm{Gua}\right]$ ions, had the same masses as those of the unmodified ODN. The masses of the $\mathrm{w}_{5}$ and $\left[\mathrm{a}_{7}-\mathrm{Gua}\right]$ ions formed from the crosslinked ODN show that the cross-linking site for this duplex ODN should not be in either the d(TATAT) or the $\mathrm{d}$ (ATATAC) portion of the ODN. Therefore, the site of MC cross-link can be assigned unambiguously to the guanine residue. Moreover, we observed the [ss + MC $+\mathrm{Gua}^{3-}$ and [ss - Gua] ${ }^{3-}$ ions (Figure 1b), which emanate from the cleavage of the glycosidic bond of the modified 2'-deoxyguanosine. The formation of the two complementary ions further supports that the guanine is involved in the formation of the interstrand crosslink.

We also acquired the product-ion spectrum of the [M $-6 \mathrm{H}]^{6-}$ ion of the above MC-cross-linked duplex ODN on an ion-trap mass spectrometer (Figure 1c). The results were similar as those obtained by SORI-CAD, i.e., we observed large mass shifts of the $\mathrm{w}_{7}{ }^{5-}, \mathrm{w}_{9}{ }^{5-}$, $\mathrm{w}_{11}{ }^{6-},\left[\mathrm{a}_{9}-\mathrm{Ade}\right]^{5-}$, and $\left[\mathrm{a}_{11}-\mathrm{Ade}\right]^{5-}$ ions. In addition, the $\mathrm{w}_{5}$ and $\left[\mathrm{a}_{7}-\mathrm{Gua}\right]$ ions again had the same masses as those formed from the unmodified ODN. Although charge state reduction by ion-ion reaction in a low resolving-power ion-trap mass spectrometer has been demonstrated to be useful for the assignments of large fragment ions [15], the ion-trap mass spectrometer without the charge reduction capability prevents us from determining the charge states of many fragment ions without ambiguity. To illustrate this, we showed a portion of the spectra containing the $\mathrm{w}_{11}{ }^{6-}$ ion (see insets in Figure $1 \mathrm{~b}$ and $\mathrm{c}$ ). The high resolving power of FT-ICR MS facilitates us to assign the charge state of the $\mathrm{w}_{11}$ ion without ambiguity. In contrast, it is difficult to establish the charge state of the same fragment from the product-ion spectrum acquired on the ion trap. Likewise, other high-mass product ions, i.e., $\mathrm{w}_{9}{ }^{5-}, \mathrm{a}_{9}-$ Ade $]^{5-}$, and $\left[\mathrm{a}_{11}-\mathrm{Ade}\right]^{5-}$ ions, could be unequivocally assigned based on SORI-CAD spectrum acquired on the FT-ICR. The charge states of those ions, however, again could not be determined from the CAD spectrum obtained on the ion trap. It is worth mentioning that here we did not take advantage of the high mass accuracy offered by the FT-ICR, the reason for which is that, with the known fragmentation behavior of deprotonated ions of ODNs in the gas phase (i.e., giving rise to the formation of the $\left[a_{n}-\right.$ base $]$ and $\mathrm{w}_{\mathrm{n}}$ ions $[14,16$, 17]), nominal masses are sufficient for us to assign the product ions.

To establish whether the above observation is general, we prepared another MC ICL-containing duplex ODN d(AAAACCGATATA)/d(TATATCGGTTTT) and subjected it to MS/MS analysis. The product-ion spectrum of the $[\mathrm{M}-5 \mathrm{H}]^{5-}$ ion $(\mathrm{m} / \mathrm{z} 1504)$ of the cross-link lesion gives $\mathrm{w}$-series product ions of $\mathrm{w}_{2}, \mathrm{w}_{4}, \mathrm{w}_{5}\left(\mathrm{w}_{5}{ }^{2-}\right)$, $\mathrm{w}_{8}{ }^{4-}, \mathrm{w}_{9}{ }^{4-}, \mathrm{w}_{10}{ }^{4-}, \mathrm{w}_{11}{ }^{5-}, w_{2}, w_{3}, w_{4}, w_{5}{ }^{2-}, w_{8}{ }^{4-}, w_{10}{ }^{4-}$, and [a - base]-series fragment ions of [a $\left.a_{3}-A d e\right],\left[a_{4}-\right.$ Ade], [a $\left.a_{5}-\mathrm{Cyt}\right],\left[\mathrm{a}_{6}-\mathrm{Cyt}\right],\left[\mathrm{a}_{7}-\mathrm{Gua}\right]\left(\left[\mathrm{a}_{7}-\mathrm{Gua}\right]^{2-}\right)$, $\left[\mathrm{a}_{10}-\mathrm{Ade}^{4-},\left[a_{4}-A d e\right],\left[a_{6}-C y t\right]\left(\left[a_{6}-C y t\right]^{2-}\right)\right.$, and $\left[a_{7}-G u a\right]\left(\left[a_{7}-G u a\right]^{2-}\right.$ ) (Figure 2a and Scheme $\mathbf{2 b}$, "Cyt" is cytosine). The " $w$ " and "[a - base $]$ " in normal and bold italic fonts represent product ions resulting from cleavages in d(AAAACCGATATA) and d(TATATCGGTTTT), respectively. Compared with the product ions emanating from cleavages of the $[\mathrm{M}-$ 




Figure 2. Product-ion spectrum of the ESI-produced $[\mathrm{M}-5 \mathrm{H}]^{5-}$ ion $(\mathrm{m} / \mathrm{z} 1504)$ of $\mathrm{d}(\mathrm{AAAAC}-$ CGATATA)/d(TATATCGGTTTT) cross-linked with MC by: (a) SORI-CAD on an FT-ICR mass spectrometer and (b) CAD on the ion-trap.

$3 \mathrm{H}]^{3-}$ ions of the single-stranded d(AAAACCGATATA) and d(TATATCGGTTTT) (Data not shown), $\mathrm{w}_{8}, \mathrm{w}_{9}, \mathrm{w}_{10}, \mathrm{w}_{11}, w_{8}, w_{10}$, and [ $\mathrm{a}_{10}-$ Ade] ions from the cross-linked ODN showed marked mass increase than the respective fragments from the cleavages of either single-stranded ODN.

Similar as the self-complementary ODN discussed above, the $\left[\mathrm{a}_{7}-\mathrm{Gua}\right]$ ion produced from the MC-crosslinked ODN had the same mass as that formed from unmodified single-stranded d(AAAACCGATATA), demonstrating that the cross-linking does not occur in the d(AAAACC) portion. In addition, the mass for the $\mathrm{w}_{5}$ ion is the same for the modified duplex ODN and the unmodified single-stranded ODN, showing that the MC is cross-linked to the d(AAAACCG) moiety. Taken together, we conclude that the guanine is the only nucleobase in d(AAAACCGATATA) that is covalently bonded with MC. Likewise, cleavages within the other strand, i.e., d(TATATCGGTTTT), gave rise to two diagnostic product ions, $\left[a_{7}-G u a\right]^{2-}$ and $w_{5}{ }^{2-}$. Both of them had the same masses as the corresponding product ions induced from the fragmentation of the singlestranded ODN. Following the similar analysis as discussed above, we determined that, of the two guanines in this strand, only the guanine at CG site is involved in cross-linking with MC. In addition, we observed the familiar $[\mathrm{ss}+\mathrm{MC}+\mathrm{Gua}]^{3-}$, , $[\mathrm{ss}-\mathrm{Gua}]^{2-},[s s+M C+$ $G u a]^{3-}$, and $[s s-G u a]^{3-}$ ions in the product-ion spectrum (Figure 2a), which supports that guanine is involved in the cross-linking with MC.

We also acquired the MS/MS of the $[\mathrm{M}-5 \mathrm{H}]^{5-}$ ion of the cross-linked ODN on the ion-trap mass spectrometer (Figure $2 b$ ). Large fragment ions, however, were again difficult to assign due to the low resolving power of the ion trap. For example, the $\mathrm{w}_{8}{ }^{4-}(\mathrm{m} / \mathrm{z}$ 1588.4) and $w_{8}{ }^{4-}(m / z 1592.9)$ ions were easily distinguishable in the SORI-CAD spectrum and their charge states were readily determined from the $\mathrm{m} / \mathrm{z}$ difference between adjacent isotope peaks. It is difficult, however, to reveal the charge states for these ions from the product-ion spectrum acquired on the ion trap. Therefore, the following results were obtained by SORI-CAD on the FTICR only.

\section{Fragmentation of Duplex ODNs Bearing a TMP Interstrand Cross-Link}

We next extended our study to ODNs bearing a $4,5^{\prime}, 8$ trimethylpsoralen (TMP) interstrand cross-link to examine whether the product-ion spectrum can also facilitate us to locate the site of interstrand cross-linking induced by TMP. The product-ion spectrum of the $[\mathrm{M}-6 \mathrm{H}]^{6-}$ ion of the TMP cross-linked d(CGCGCTAGCGCG) (Figure 3a) again showed cleavages within either strand to give $\mathrm{w}$-series products of $\mathrm{w}_{1}, \mathrm{w}_{2}, \mathrm{w}_{3}, \mathrm{w}_{4}{ }^{2-}, \mathrm{w}_{5}{ }^{2-}, \mathrm{w}_{7}{ }^{5-}, \mathrm{w}_{9}{ }^{5-}$, 


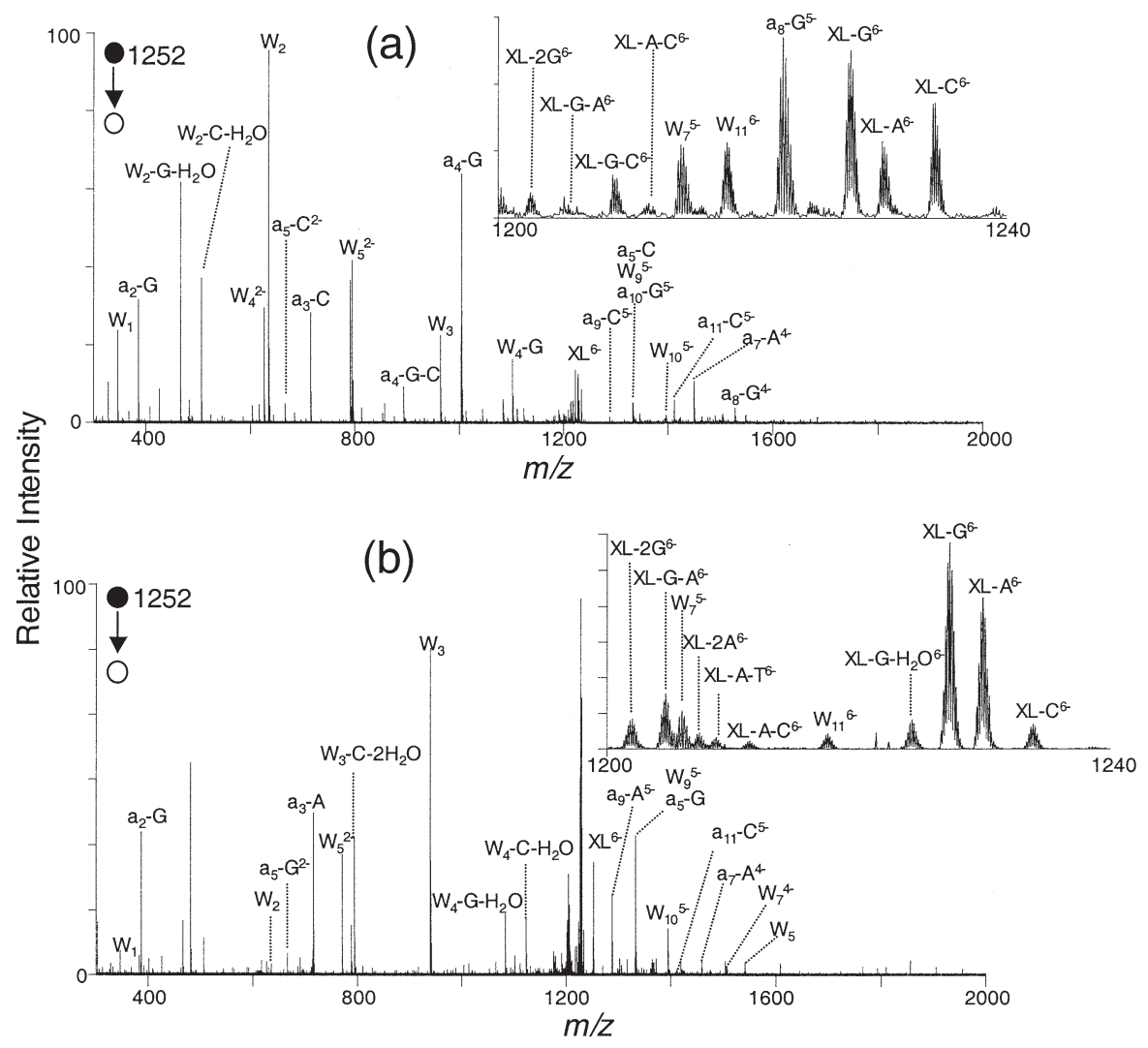

Figure 3. Product-ion spectrum of the ESI-produced $[\mathrm{M}-6 \mathrm{H}]^{6-}$ ion $(\mathrm{m} / \mathrm{z} 1252)$ of double-stranded, self-complementary ODNs d(CGCGCTAGCGCG) (a) and d(CGATGTACATCG) (b) cross-linked with TMP. The spectra were acquired by SORI-CAD on the FT-ICR.

$\mathrm{w}_{10}{ }^{5-}, \mathrm{w}_{11}{ }^{6-}$ and $[\mathrm{a}-$ base $]$-series fragments of $\left[\mathrm{a}_{2}-\right.$ Gua], [a $a_{3}-$ Cyt $],\left[a_{4}-\right.$ Gua], $\left[a_{5}-\right.$ Cyt $]\left(\left[a_{5}-C y t\right]^{2-}\right),\left[a_{7}\right.$ - Ade $^{4-},\left[\mathrm{a}_{8}-\mathrm{Gua}\right]^{5-},\left[\mathrm{a}_{9}-\mathrm{Cyt}\right]^{5-},\left[\mathrm{a}_{10}-\mathrm{Gua}\right]^{5-}$ (Scheme 2c). Among these fragment ions, $\mathrm{w}_{7}, \mathrm{w}_{9}, \mathrm{w}_{10}, \mathrm{w}_{11}$, $\left[\mathrm{a}_{7}-\right.$ Ade], [a $\left.\mathrm{a}_{8}-\mathrm{Gua}\right],\left[\mathrm{a}_{9}-\mathrm{Cyt}\right]$, and $\left[\mathrm{a}_{10}-\mathrm{Gua}\right]$ from the TMP cross-linked ODN exhibited higher masses than the corresponding fragments of the single-stranded ODN. For example, the mass of the $\left[\mathrm{a}_{7}-\right.$ Ade] ion from the cross-linked ODN shows that it bears the remnant of the strand being cleaved, the TMP moiety, and the intact opposite single strand, showing that the cross-linking occurs in the d(CGCGCT) portion. Similarly, the $\mathrm{w}_{7}$ ion has a much higher mass than the corresponding fragment from the unmodified ODN, illustrating that cross-linking can only be in the d(TAGCGCG) moiety. Taken together, the thymidine residue is involved in cross-linking with the TMP.

The ODN we just discussed contains only one thymine, we then examined a duplex ODN with three thymines in each strand, one at TA site and another two at AT sites, i.e., d(CGATGTACATCG). We chose this substrate to demonstrate that the product-ion spectrum can allow us to establish, among the three thymines, which is involved in the formation of the covalent bond with TMP. Figure $3 b$ is the product-ion spectrum of the $[\mathrm{M}-6 \mathrm{H}]^{6-}$ ion of a self-complementary duplex ODN $\mathrm{d}$ (CGATGTACATCG) that is cross-linked with a TMP.
Again, a series of $\mathrm{w}$ and [a - base] ions were formed upon SORI-CAD, among them $\mathrm{w}_{7}{ }^{5-}, \mathrm{w}_{9}{ }^{5-}, \mathrm{w}_{10}{ }^{5-}$, $\mathrm{w}_{11}{ }^{6-},\left[\mathrm{a}_{7}-\mathrm{Ade}\right]^{4-},\left[\mathrm{a}_{9}-\mathrm{Ade}\right]^{5-}$, and $\left[\mathrm{a}_{11}-\mathrm{Cyt}\right]^{5-}$ (Scheme 2d) from this TMP cross-linked duplex ODN showed higher masses than the respective fragments from the cleavages of the $[\mathrm{M}-3 \mathrm{H}]^{3-}$ ion of the unmodified single-stranded ODN. The higher masses of the $\mathrm{w}_{7}$ and $\left[\mathrm{a}_{7}-\mathrm{Ade}\right]^{4-}$ ions formed from the cross-linked ODN than from the corresponding singlestranded ODN demonstrate that the cross-linking occurs in the overlapping region of $\mathrm{d}$ (CGATGT) and $\mathrm{d}$ (TACATCG). Therefore, only the thymine base at the TA site is covalently bonded to the TMP, which is in line with what has been reported in the literature [3].

\section{Conclusions}

Mass spectrometry has become a powerful tool for examining large biomolecule complexes in solution and tandem mass spectrometry is widely used for gaining structural information of these macromolecular complexes. Here we showed that the fragmentation of interstrand cross-linked duplex ODN is analogous to that of a single-stranded ODN bearing a modified nucleobase [18-21]. The major difference between the two is that the interstrand cross-linking results in much larger mass increase of the nucleobase that is being 
cross-linked. Therefore, fragment ions containing the modified nucleobase are much larger than the respective product ions from the cleavage of the corresponding unmodified ODN. The mass shifts of those product ions provide an opportunity for locating the sites of the interstrand cross-linking by MS/MS. These product ions, however, are multiply-charged in ESI-MS/MS, which necessitate the application of a high resolving power instrument for their assignments. As demonstrated in this paper, product-ion spectra acquired by SORI-CAD on an FT-ICR mass spectrometer allow us to assign readily the fragment ions, which further enable us to establish the sites of MC or TMP interstrand cross-linking in medium-size ODNs, i.e., 12-mer. We also observed that collisional activation of MC ICLbearing duplex ODNs gave rise to cleavages of the glycosidic bond of the modified 2'-deoxyguanosine to give a complementary pair of fragment ions, [ss - Gua] and [ss $+\mathrm{MC}+$ Gua], which resulted from the cleavage of the glycosidic bond of the modified 2'-deoxyguanosine. The formation of these ions allowed us to establish that the guanine base is involved in the cross-linking. Similar cleavage of the glycosidic bond of the TMP cross-linked thymidine residue does not occur, which is likely due to the low proton affinity of the modified thymine [17].

It is of note that the product-ion spectra for crosslinked non-self-complementary ODNs are complicated. Once the sequences for the ODNs are known, the sites of the cross-link, however, can be readily determined from the mass shifts of product ions as stated above. The method, therefore, will be useful for examining the sequence-selective reactivities of ICL drugs with medium-sized ODNs. In addition, this method is complementary to the one that we reported previously [7], in which structure elucidation of DNA interstrand crosslink was achieved by a combination of nuclease P1 digestion with mass spectrometry. Although MC-ICL and TMP-ICL were employed as model systems for this and previous studies, we anticipate that the methods described should also be useful for elucidating the structure and locating the sites of interstrand cross-links formed between ODNs and other agents.

\section{Acknowledgments}

The authors thank the National Institute of Health for supporting this research (R01 CA96906).

\section{References}

1. Rajski, S. R.; Williams, R. M. DNA Cross-Linking Agents as Antitumor Drugs. Chem. Rev. 1998, 98, 2723-2796.

2. Luce, R. A.; Hopkins, P. B. Chemical Cross-Linking of Drugs to DNA. Methods Enzymol. 2001, 340, 396-412.

3. Kanne, D.; Straub, K.; Hearst, J. E.; Rapoport, H. Isolation and Characterization of Pyrimidine-Psoralen-Pyrimidine Photodiadducts from DNA. J. Am. Chem. Soc. 1982, 104, 6754-6764.
4. Tomasz, M.; Lipman, R.; Chowdary, D.; Pawlak, J.; Verdine, G. L.; Nakanishi, K. Isolation and Structure of a Covalent Cross-Link Adduct Between Mitomycin $C$ and DNA. Science 1987, 235, 1204-1208.

5. Weidner, M. F.; Millard, J. T.; Hopkins, P. B. Determination at Single Nucleotide Resolution of the Sequence Specificity of DNA Interstrand Cross-Linking Agents in DNA Fragments. J. Am. Chem. Soc. 1989, 111, 9270-9272.

6. Weidner, M. F.; Sigurdsson, S. T.; Hopkins, P. B. Sequence Preferences of DNA Interstrand Cross-Linking Agents: dGto-dG Cross-Linking at 5'-CG by Structurally Simplified Analogues of Mitomycin C. Biochemistry 1990, 29, 9225-9233.

7. Wang, Y.; Wang, Y. Structure Elucidation of DNA Interstrand Cross-Link by a Combination of Nuclease P1 Digestion with Mass Spectrometry. Anal. Chem. 2003, 75, 6306-6313.

8. Marshall, A. G.; Hendrickson, C. L.; Jackson, G. S. Fourier Transform Ion Cyclotron Resonance Mass Spectrometry: A Primer. Mass Spectrom. Rev. 1998, 17, 1-35.

9. Amster, I. J. Fourier Transform Mass Spectrometry. J. Mass Spectrom. 1996, 31, 1325-1337.

10. Yeung, A. T.; Jones, B. K.; Chu, C. T. Photoreactivities and Thermal Properties of Psoralen Cross-Links. Biochemistry 1988, 27, 3204-3210.

11. Borowy-Borowski, H.; Lipman, R.; Chowdary, D.; Tomasz, M. Duplex Oligodeoxyribonucleotides Cross-Linked by Mitomycin $C$ at a Single Site: Synthesis, Properties, and Cross-Link Reversibility. Biochemistry 1990, 29, 2992-2999.

12. Gauthier, J. W.; Trautman, T. R.; Jacobson, D. B. Sustained Off-Resonance Irradiation for Collision-Activated Dissociation Involving Fourier Transform Mass Spectrometry. CollisionActivated Dissociation Technique that Emulates Infrared Multiphoton Dissociation. Anal. Chim. Acta 1991, 246, 211-225.

13. Senko, M. W.; Speir, J. P.; McLafferty, F. W. Collisional Activation of Large Multiply Charged Ions Using Fourier Transform Mass Spectrometry. Anal. Chem. 1994, 66, 28012808.

14. McLuckey, S. A.; Van Berkel, G. J.; Glish, G. L. Tandem Mass Spectrometry of Small, Multiply Charged Oligonucleotides. J. Am. Soc. Mass Spectrom. 1992, 3, 60-70.

15. Stephenson, Jr.; J. L., McLuckey, S. A. Simplification of Product Ion Spectra Derived from Multiply Charged Parent Ions via Ion/Ion Chemistry. Anal. Chem. 1998, 70, 3533-3544.

16. McLuckey, S. A.; Habibi-Goudarzi, S. Decompositions of Multiply Charged Oligonucleotide Anions. J. Am. Chem. Soc. 1993, 115, 12085-12095.

17. Wang, Z.; Wan, K. X.; Ramanathan, R.; Taylor, J. S.; Gross, M. L. Structure and Fragmentation Mechanisms of Isomeric T-Rich Oligodeoxynucleotides: A Comparison of Four Tandem Mass Spectrometric Methods. J. Am. Soc. Mass Spectrom. 1998, 9, 683-691.

18. Stemmler, E. A.; Buchanan, M. V.; Hurst, G. B.; Hettich, R. L. Analysis of Modified Oligonucleotides by Matrix-Assisted Laser Desorption/Ionization Fourier Transform Mass Spectrometry. Anal. Chem. 1995, 67, 2924-2930.

19. Marzilli, L. A.; Wang, D.; Kobertz, W. R.; Essigmann, J. M.; Vouros, P. Mass Spectral Identification and Positional Mapping of Aflatoxin B1-Guanine Adducts in Oligonucleotides. J. Am. Soc. Mass Spectrom. 1998, 9, 676-682.

20. Iannitti, P.; Sheil, M. M.; Wickham, G. High Sensitivity and Fragmentation Specificity in the Analysis of Drug-DNA Adducts by Electrospray Tandem Mass Spectrometry. J. Am. Chem. Soc. 1997, 119, 1490-1491.

21. McLuckey, S. A.; Habibi-Goudarzi, S. Ion Trap Tandem Mass Spectrometry Applied to Small Multiply Charged Oligodeoxyribonucleotides with a Modified Base. J. Am. Soc. Mass Spectrom. 1994, 5, 740-747. 